\title{
Obeticholic acid for PBC
}

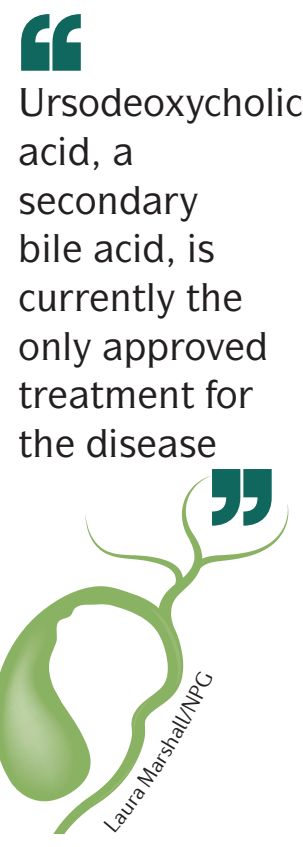

Results from a new clinical trial show that obeticholic acid decreases serum alkaline phosphatase and bilirubin levels in patients with primary biliary cholangitis (PBC, previously known as primary biliary cirrhosis). However, treatment was associated with increased risk of pruritis in patients receiving higher doses of the drug. $\mathrm{PBC}$ is an autoimmune liver disease characterized by progressive destruction of bile ducts, resulting in cirrhosis and end-stage liver disease. Ursodeoxycholic acid, a secondary bile acid, is currently the only approved treatment for the disease; the lack of therapies for patients not tolerating or responding to this drug has driven investigation of the semi-synthetic bile acid obeticholic acid. However, its use, particularly at doses $>10 \mathrm{mg}$ per day, had been associated with severe pruritis in phase II trials.
To address whether obeticholic acid could improve $\mathrm{PBC}$ in patients with intolerance or inadequate response to ursodeoxycholic acid, researchers conducted a phase III, randomized, double-blind placebo-controlled trial. Either as monotherapy or in addition to ursodeoxycholic acid, obeticholic acid was given at an initial dose of $5 \mathrm{mg}$ (and up-titrated to $10 \mathrm{mg}$ as necessary) or $10 \mathrm{mg}$ per day. The primary composite end point at 12 months was a serum alkaline phosphatase level $<1.67$-fold the upper limit of normal (and a reduction of $\geq 15 \%$ from baseline), and a serum bilirubin level at or below the upper limit of normal. Both parameters are validated biomarkers of outcomes in patients with $\mathrm{PBC}$.

$46 \%$ of patients in the $5-10 \mathrm{mg}$ group $(n=71)$ and $47 \%$ of patients in the $10 \mathrm{mg}$ group $(n=73)$ achieved the primary end point, compared with just $10 \%$ of the placebo group $(n=73 ; P<0.001$ for both comparisons).

"In the 5-10 mg group, around 50\% stayed under the $5 \mathrm{mg}$ dose, and the dropout after 1 year due to pruritus was only $1 \%$ in this arm", explains author Frederik Nevens. By contrast, dropout rate at 1 year was $10 \%$ in the $10 \mathrm{mg}$ group. Notably, $97 \%$ of patients in the 5-10 mg group opted to continue treatment during an open-label extension phase at the end of the blinded study. Nevens and colleagues are following-up patients in this study for 5 years to assess long-term tolerance of obeticholic acid and its effect on patients with advanced liver disease (18-20\% of the baseline study population).

Hugh Thomas

ORIGINAL ARTICLE Nevens, F. et al. A placebocontrolled trial of obeticholic acid in primary biliary cholangitis. N. Engl.J. Med. 375, 631-643 (2016) 